\title{
Seminare 2014
}

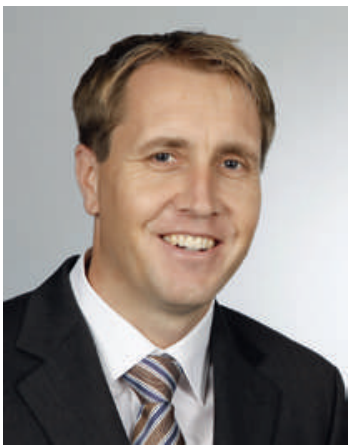

René Häller, Geschäftsführer FMH Consulting Services

\section{Praxiseröffnung/-übernahme}

Das Seminar richtet sich an Ärztinnen und Ärzte, welche vor einer Praxiseröffnung (Einzel-/Gruppenpraxis), dem Einstieg in eine Gruppenpraxis oder vor einer Praxisübernahme stehen.

\section{Themen}

- Juristische Aspekte (Praxisbewilligung, Zulassung zur Sozialversicherung, Vertragswesen)

- Gesellschaftsformen/Ehe- und Erbrecht (Privat-/Geschäftsvermögen, Güterstand, Erbschaftsplanung)

- Praxiseinrichtung (Inneneinrichtung, Kostenberechnung)

- Praxisadministration (Leistungserfassungsund Abrechnungssysteme)

- Bewertung einer Arztpraxis (Berechnung Inventarwert und Goodwill als Verhandlungsbasis)

- Finanzierung der Arztpraxis (Businessplan, Kredite, Absicherungsmöglichkeiten)

- Versicherungen/Vorsorge/Vermögen (Personen- und Sachversicherungen, Vorsorgeplanung).

\section{Sponsoren}

Die Kosten werden durch diverse Sponsoren (siehe www.fmhservices.ch) gedeckt.

\begin{tabular}{|c|c|c|}
\hline \multicolumn{3}{|c|}{ Daten } \\
\hline К01 & $\begin{array}{l}\text { Donnerstag, } \\
\text { 13. März } 2014 \\
\text { 9.00-16.30 Uhr }\end{array}$ & Zürich FMT \\
\hline K02 & $\begin{array}{l}\text { Donnerstag, } \\
\text { 8. Mai } 2014 \\
\text { 16.00-20.30 Uhr }\end{array}$ & $\begin{array}{l}\text { St. Gallen } \\
\text { Hotel Einstein }\end{array}$ \\
\hline K03 & $\begin{array}{l}\text { Donnerstag, } \\
\text { 5. Juni } 2014 \\
9.00-16.30 \text { Uhr }\end{array}$ & $\begin{array}{l}\text { Bern } \\
\text { Schmiedstube }\end{array}$ \\
\hline
\end{tabular}

Obwohl rund $40 \%$ aller Ärztinnen und Ärzte früher oder später eine eigene Arzt-/Gruppenpraxis führen und nebst dem medizinischen Know-how auch jenes eines Unternehmers innehaben sollten, wird im Verlauf des Medizinstudiums die Thematik der Unternehmensführung nie oder nur kurz fokussiert. Die FMH Services, welche sich um die betriebswirtschaftlichen Angelegenheiten ihrer Genossenschafter kümmern, schliessen diese Lücke und bieten dazu spezifische Seminare an. In diesen Seminaren werden den Ärztinnen und Ärzten Grundlagen vermittelt für den Einstieg in die eigene Arzt-/Gruppenpraxis, deren Führung und deren optimale Übergabe an einen/eine Nachfolger/in. Die Ärztinnen und Ärzte werden auf den Umgang und die Zusammenarbeit mit Behörden sowie Fachspezialisten, wie Treuhänder, Versicherungs- und Finanzberater, Kreditberater bei Banken, Juristen, Architekten usw., vorbereitet. Die einzelnen Themen werden von ausgewiesenen Experten der FMH Services referiert.

\begin{tabular}{|lll}
\hline K04 & $\begin{array}{l}\text { Donnerstag, } \\
\text { 4. September 2014 } \\
\text { 9.00-16.30 Uhr }\end{array}$ & Zürich FMT \\
\hline K05 & $\begin{array}{l}\text { Donnerstag, } \\
\text { 6. November 2014 } \\
\text { 9.00-16.30 Uhr }\end{array}$ & $\begin{array}{l}\text { Basel } \\
\text { Hotel Victoria }\end{array}$ \\
\hline
\end{tabular}

Praxisübergabe

Das Seminar richtet sich an zukünftige Praxisübergeber/innen. Idealtermin: 5-10 Jahre vor geplanter Übergabe (aus steuer- und vorsorgeplanerischen Gründen).

\section{Themen}

- Juristische Aspekte (Praxisübergabevertrag, allg. Vertragswesen, Übergabe der Krankengeschichten)

- Nachfolgeplanung und Bewertung einer Arztpraxis (projektorientiertes Vorgehen in der Nachfolgeplanung, Berechnung Inventarwert und Goodwill als Verhandlungsbasis)

- Versicherungen/Vorsorge/Vermögen (Übergabe/Auflösung von Versicherungsverträgen, Pensions- und Finanzplanung)

- Steuern (Steueraspekte bei der Praxisübergabe: Optimierung der steuerlichen Auswirkungen, Liquidations- und Grundstückgewinnsteuer, Bestimmung des optimalen Übergabezeitpunktes).

\section{Sponsoren}

Die Kosten werden durch diverse Sponsoren (siehe www.fmhservices.ch) gedeckt.

\begin{tabular}{|c|c|c|}
\hline \multicolumn{3}{|c|}{ Daten } \\
\hline K06 & $\begin{array}{l}\text { Donnerstag, } \\
\text { 20. März } 2014 \\
\text { 13.30-18.00 Uhr }\end{array}$ & Zürich FMT \\
\hline K07 & $\begin{array}{l}\text { Donnerstag, } \\
\text { 22. Mai } 2014 \\
\text { 16.00-20.30 Uhr }\end{array}$ & $\begin{array}{l}\text { St. Gallen } \\
\text { Hotel Einstein }\end{array}$ \\
\hline
\end{tabular}

\begin{tabular}{lll} 
K08 & $\begin{array}{l}\text { Donnerstag, } \\
\text { 12. Juni 2014 } \\
\text { 13.30-18.00 Uhr }\end{array}$ & $\begin{array}{l}\text { Bern } \\
\text { Schmiedstube }\end{array}$ \\
\hline K09 & $\begin{array}{l}\text { Donnerstag, } \\
\text { 11. September 2014 } \\
\text { 13.30-18.00 Uhr }\end{array}$ & Zürich FMT \\
\hline K10 & $\begin{array}{l}\text { Donnerstag, } \\
\text { 13. November 2014 } \\
\text { 13.30-18.00 Uhr }\end{array}$ & $\begin{array}{l}\text { Basel } \\
\text { Hotel Victoria }\end{array}$ \\
\hline
\end{tabular}

Finanz- und Steuerplanung

Das Seminar richtet sich an Praxiseröffner/innen, Praxisübernehmer/innen sowie an bereits praxistätige Ärztinnen und Ärzte.

\section{Themen}

- Finanzplanung (Businessplan, buchhalterische Massnahmen vor Praxiseröffnung/ -übernahme, Standardkontenplan, doppelte Buchhaltung, EDV-unterstützte Buchführungslösung)

- Steuern (Steueraspekte bei Eintritt in die Selbständigkeit, Steuerfallen und Steuerrisiken, optimierte Steuerplanung).

\section{Kosten}

Für FMH Services-Mitglieder kostenlos.

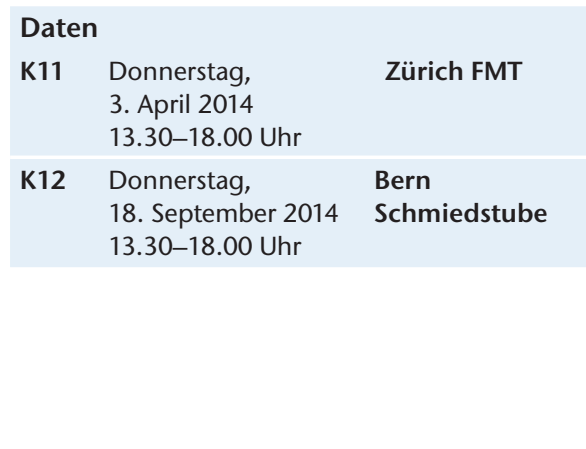




\section{Praxiscomputerworkshop}

Der Workshop richtet sich an praxiseröffnende sowie an bereits praxistätige Ärztinnen und Ärzte.

Inhalt

- Anforderungen an ein Praxisinformationssystem (Einführung)

- Evaluationsprozess (projektorientiertes Vorgehen in der Evaluation eines Praxisinformationssystems)

- Präsentation von sechs führenden Praxisinformationssystemen (Leistungserfassung, elektronisches Abrechnen unter Einbezug der TrustCenter, Agendaführung, Statistiken, Laborgeräteeinbindung, elektronische Krankengeschichte, Finanzbuchhaltungslösungen usw.).

\begin{tabular}{|c|c|c|}
\hline \multicolumn{3}{|c|}{ Daten } \\
\hline K13 & $\begin{array}{l}\text { Donnerstag, } \\
\text { 27. März } 2014 \\
\text { 13.30-18.00 Uhr }\end{array}$ & $\begin{array}{l}\text { Zürich } \\
\text { Technopark }\end{array}$ \\
\hline K14 & $\begin{array}{l}\text { Donnerstag, } \\
\text { 3. Juli } 2014 \\
\text { 13.30-18.00 Uhr }\end{array}$ & $\begin{array}{l}\text { Bern } \\
\text { BERNEXPO }\end{array}$ \\
\hline K15 & $\begin{array}{l}\text { Donnerstag, } \\
\text { 27. November } 2014 \\
\text { 13.30-18.00 Uhr }\end{array}$ & $\begin{array}{l}\text { Olten } \\
\text { Stadttheater }\end{array}$ \\
\hline
\end{tabular}

\section{Gruppenpraxis}

Das Seminar richtet sich an in Ausbildung stehende Ärztinnen und Ärzte, die sich einer Gruppenpraxis anschliessen wollen, sowie an praxistätige Ärztinnen und Ärzte, die ihre Einzelpraxis an eine Gruppenpraxis anschliessen wollen.

\section{Themen}

- Strategie (Ziele der Gruppenpraxis; Gestaltung des Angebots)

- Unternehmer (Zusammensetzung des Teams; Verhaltensregeln, Finanzielle Beteiligung und Entschädigungsmodelle)

- Finanzen und Recht (Versicherung, Vorsorge und Vermögen; Rechtsform, Finanzen, Steuern)

- Standort, Praxisobjekt (Anforderungen an Standort; Konkurrenzanalyse, Praxiseinrichtung, Kosten)

- Personal (Qualifikationen; Gesetze, Reglemente, Verträge)

- Führung und Organisation (Struktur und Abläufe; Aufgaben, Verantwortungen, Kompetenzen)

- EDV und Administration (Anforderungen an Praxisinformatik; Evaluation)

- Praxisbericht (Erfahrungsbericht eines Arztes - Mitgründer einer Gruppenpraxis aus der Region)

\section{Sponsoren}

Die Kosten werden durch diverse Sponsoren (siehe www.fmhservices.ch) gedeckt.

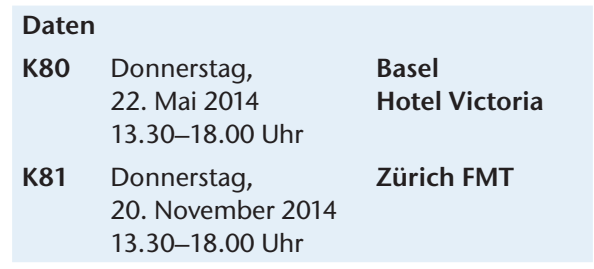

Röntgen in der Arztpraxis

Das Seminar richtet sich an Ärztinnen und Ärzte mit bestehender Praxis und an solche, die vor einer Praxiseröffnung/-übernahme stehen.

\section{Themen}

- Rentabilität Röntgen in der Arztpraxis

- Evaluation und Beschaffung neuer oder gebrauchter Anlagen

- Möglichkeiten der Umrüstung von analogen zu digitalen Anlagen

- Vor- und Nachteile analoger und digitaler Systeme

- Komplette Marktübersicht mit Preisen und Leistungskomponenten.

\section{Kosten}

Für FMH Services-Mitglieder kostenlos.

Datum
$\begin{array}{lll}\text { K16 } & \begin{array}{l}\text { Donnerstag, } \\ \text { 28. August 2014 }\end{array} & \begin{array}{l}\text { Niederscherli } \\ \text { WIROMA AG }\end{array} \\ & \text { W9.30-16.00 Uhr } & \end{array}$

Tarifwerk TARMED - Einführungskurs Das Seminar richtet sich an Ärztinnen und Ärzte mit bestehender Praxis und an solche, die kurz vor einer Praxiseröffnung oder Praxisübernahme stehen. Der Einführungskurs vermittelt den Kursteilnehmern die Grundlagen des Tarifwerkes TARMED.

\section{Themen}

- Fakten (gesetzliche und vertragliche Grundlagen)

- Struktur (Tarifbrowser, Grundstruktur, Regelhierarchie, Leistungsblöcke, Leistungsgruppen)

- Generelle Interpretationen («Allgemeine Grundleistungen», «Hauptleistungen, Zuschlagsleistungen», «Nichtärztliche Leistungserbringung» usw.)

- Parameter einer Tarifposition («Quantitative und Qualitative Dignität», «Ärztliche Leistung AL», «Assistenz», «Raumbelegung» usw.)

- Tarifpositionen aus dem Kapitel 00 Grundleistungen

- Praxislabor und Präsenzdiagnostik (neue Analyseliste)

- Organisationen und Informationsquellen.

Kosten

200 CHF (inkl. Kursunterlagen).

\begin{tabular}{|lll}
\multicolumn{2}{c}{ Daten } \\
K60 & $\begin{array}{l}\text { Dienstag, Olten } \\
\text { 18. März 2014 } \\
\text { 14.00-17.15 Uhr }\end{array}$ & Hotel Arte \\
K61 & $\begin{array}{l}\text { Dienstag, } \\
\text { 13. Mai 2014 } \\
\text { 14.00-17.15 Uhr }\end{array}$ & $\begin{array}{l}\text { Olten } \\
\text { Hotel Arte }\end{array}$ \\
\hline K62 & $\begin{array}{l}\text { Dienstag, } \\
\text { 16. September 2014 } \\
\text { 14.00-17.15 Uhr }\end{array}$ & $\begin{array}{l}\text { Olten } \\
\text { Hotel Arte }\end{array}$ \\
\hline
\end{tabular}

\section{Praxismarketing für Ärzte}

Das Seminar richtet sich an Ärztinnen und Ärzte, welche vor einer Praxiseröffnung stehen oder bereits eine Praxis führen.

\section{Themen}

- Service und Kundenorientierung: Aus einer Arztpraxis wird DIE Praxis.

- Menschen überraschen und hohes Nveau halten

- Telefon, Empfang, Teamentwicklung

- Werbemöglichkeiten.

\section{Kosten}

Für FMH Services-Mitglieder kostenlos.

$\begin{array}{lll}\text { Daten } & & \\ \text { K63 } & \begin{array}{l}\text { Donnerstag, } \\ \text { 20. März 2014 } \\ \text { 13.30-18.00 Uhr }\end{array} & \begin{array}{l}\text { Lachen Prama } \\ \text { Marketing \& } \\ \text { Vertriebs GmbH }\end{array} \\ \text { K64 } & \begin{array}{l}\text { Mittwoch, } \\ \text { 12. November 2014 } \\ \text { 13.30-18.00 Uhr }\end{array} & \begin{array}{l}\text { Lachen PRAMA } \\ \text { Marketing \& } \\ \text { Vertriebs GmbH }\end{array}\end{array}$

Telefonseminar für MPAs

(bzw. Praxisteam)

Das Seminar richtet sich an MPAs mit telefonischem Kundenkontakt sowie Auszubildende, die zum professionellen Telefonieren angeleitet werden sollen.

\section{Themen}

- Die Medizinische Praxisassistentin als Visitenkarte der Praxis

- Image der Arztpraxis. MPAs repräsentieren die Unternehmenskultur, organisieren die Praxis und sind somit ein wesentlicher Bestandteil für den Unternehmenserfolg.

- Bedeutung des ersten Telefonkontakts

- Richtig telefonieren - eine Anleitung.

\section{Kosten}

300 CHF (inkl. sämtlicher Kursunterlagen und Verpflegungen).

Praxisteams erhalten einen Rabatt von 20\% pro Teilnehmer/in. 


\begin{tabular}{lll}
$\begin{array}{l}\text { Daten } \\
\text { K65 }\end{array}$ & $\begin{array}{l}\text { Mittwoch, } \\
\text { 26. März 2014 } \\
\text { 09.00-16.30 Uhr }\end{array}$ & Zürich FMT \\
K66 & $\begin{array}{l}\text { Mittwoch, } \\
\text { 5. November 2014 } \\
\text { 09.00-16.30 Uhr }\end{array}$ & $\begin{array}{l}\text { Bern } \\
\text { Schmiedstube }\end{array}$ \\
\hline
\end{tabular}

Kommunikation mit Patienten für MPAs (bzw. Praxisteam)

Das Seminar richtet sich an MPAs sowie Auszubildende, die zu einer professionelleren Kommunikation mit Patienten (auch schwierigen) angeleitet werden sollen und sich in der Kommunikation im Team verbessern möchten.

\section{Themen}

- kommunikative Techniken (aktives Zuhören, 4-Ohren-Prinzip)

- Kundenorientierte Kommunikation

- eigenes Konflikt-Verhalten

- positiver Umgang mit Reklamationen und schwierigen Gesprächssituationen.

\section{Kosten}

300 CHF (inkl. sämtlicher Kursunterlagen und Verpflegungen).

Praxisteams erhalten einen Rabatt von 20\% pro Teilnehmer/in.

\begin{tabular}{|c|c|c|}
\hline \multicolumn{3}{|c|}{ Daten } \\
\hline K67 & $\begin{array}{l}\text { Mittwoch, } \\
\text { 19. März } 2014 \\
\text { 09.00-16.30 Uhr }\end{array}$ & Zürich FMT \\
\hline K68 & $\begin{array}{l}\text { Mittwoch, } \\
\text { 10. September } 2014 \\
09.00-16.30 \text { Uhr }\end{array}$ & $\begin{array}{l}\text { Bern } \\
\text { Schmiedstube }\end{array}$ \\
\hline
\end{tabular}

\section{Crashkurs Versicherungsmedizin}

Das Seminar richtet sich an Ärztinnen und Ärzte, vom Assistenzarzt bis zum Klinikdirektor, vom Grundversorger bis zum Superspezialisten. Das Seminar wird in Zusammenarbeit mit der Schweiz. Gesellschaft der Vertrauensund Versicherungsärzte (SGV) durchgeführt.

\section{Themen}

- Spielregeln der Versicherungsmedizin von KVG, UVG und IVG anhand von Fallbeispielen

- Gesetze und Verordnungen

- Zuständige Ämter.

\section{Kosten}

150 CHF (inkl. sämtlicher Kursunterlagen und Verpflegungen).

\begin{tabular}{|lll}
\multicolumn{2}{l}{ Daten } \\
K90 & $\begin{array}{l}\text { Donnerstag, } \\
\text { 27. März 2014 } \\
\text { 13.30-16.00 Uhr }\end{array}$ & $\begin{array}{l}\text { Bern } \\
\text { Schmiedstube }\end{array}$ \\
\hline K91 & $\begin{array}{l}\text { Donnerstag, } \\
\text { 28. August 2014 } \\
\text { 13.30-16.00 Uhr }\end{array}$ & Zürich FMT \\
\hline
\end{tabular}

\section{Erfolgreich in die Pension}

Das Seminar richtet sich an Ärztinnen und Ärzte, welche die Pensionierung (auch eine frühzeitige) nicht dem Zufall überlassen wollen.

\section{Inhalt}

Reich in Rente?

- Kapitalbedarf

- Vorsorgelösungen

- Steuerlich optimiertes Vorgehen
Treuhand und Altersvorsorge?

- Praxisverkauf in Abstimmung mit Altersplanung

- Möglichkeiten der Steuereinsparung

Praxisbeispiele

- Planung der Altersvorsorge.

\section{Kosten}

100 CHF (inkl. sämtlicher Kursunterlagen und Verpflegung).

\begin{tabular}{|c|c|c|}
\hline \multicolumn{3}{|c|}{ Daten } \\
\hline K30 & $\begin{array}{l}\text { Donnerstag, } \\
\text { 26. Juni } 2014 \\
\text { 18.00-20.30 Uhr }\end{array}$ & Zürich FMT \\
\hline K31 & $\begin{array}{l}\text { Donnerstag, } \\
\text { 25. September } 2014 \\
18.00-20.30 \mathrm{Ur}\end{array}$ & $\begin{array}{l}\text { Basel } \\
\text { Hotel Victoria }\end{array}$ \\
\hline
\end{tabular}

\section{Anmeldung und Auskunft}

www.fmhservices.ch oder FMH Consulting Services, Cornelia Fuchs, Burghöhe 1, 6208 Oberkirch, Tel. 04192500 77, Fax 0419210586

\section{Hinweis}

Bei sämtlichen Seminaren, bei denen die Kosten teilweise oder gänzlich von Seminarsponsoren gedeckt werden, werden die Teilnehmeradressen den jeweiligen Sponsoren zur Verfügung gestellt.

\section{Annullierungsbedingungen}

Bei Abmeldungen oder Fernbleiben werden folgende Unkostenbeiträge erhoben:

- 50 CHF pro Person ab 14 Tage vor Seminarbeginn;

- 100 CHF pro Person ab 7 Tage vor Seminarbeginn oder Fernbleiben.

\section{Séminaires 2014}

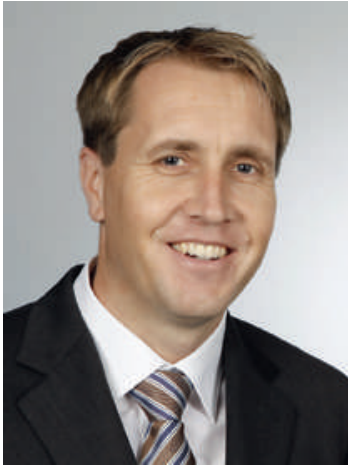

René Häller,

Directeur FMH Consulting Services

\section{Ouverture et reprise d'un cabinet} médical

Le séminaire est destiné aux médecins sur le point d'ouvrir un cabinet médical (individuel ou de groupe), de joindre un cabinet de groupe ou de reprendre un cabinet existant.

Bien qu'environ $40 \%$ de tous les médecins ouvrent tôt ou tard leur propre cabinet médical et ont besoin de connaissances en gestion d'entreprise hormis leur savoir-faire médical, cette thématique n'est souvent pas ou peu abordée. FMH Services qui s'occupe de la gestion d'entreprise de ses membres, comble désormais cette lacune en proposant des séminaires spécifiques. Dans ces séminaires, les médecins peuvent acquérir les bases nécessaires pour ouvrir leur propre cabinet médical ou un cabinet de groupe ou pour remettre leur cabinet de manière optimale. Les médecins obtiennent également des conseils sur la manière de collaborer avec les autorités ou avec des spécialistes tels que des experts-comptables, des conseillers financiers, en assurance ou en crédits bancaires, des juristes, des architectes, etc. Ces différents thèmes sont abordés par des experts qualifiés de FMH Services.

\section{Contenu}

- Business plan (préparation du plan de financement et crédit d'exploitation, financement par la banque)

- Aménagement (implantation, projet et concept d'aménagement, choix du mobilier, budget)

- Estimation d'un cabinet (inventaire et goodwill)

- Laboratoire

- Administration d'un cabinet medical
- Assurances

- Passage du statut de salarié à celui d'indépendant

- Fiscalité

\section{Sponsors}

Les coûts sont pris en charge par divers sponsors (voir www.fmhservices.ch). 


\begin{tabular}{|lll}
\hline $\begin{array}{l}\text { Dates } \\
\text { K20 }\end{array}$ & $\begin{array}{l}\text { Jeudi } \\
13 \text { mars 2014 } \\
\text { 13.30-18.00 h }\end{array}$ & $\begin{array}{l}\text { Lausanne } \\
\text { World Trade } \\
\text { Center }\end{array}$ \\
\hline K21 & $\begin{array}{l}\text { Jeudi } \\
8 \text { mai 2014 } \\
13.30-18.00 ~ h\end{array}$ & $\begin{array}{l}\text { Genève } \\
\text { Crowne Plaza }\end{array}$ \\
K22 & $\begin{array}{l}\text { Jeudi } \\
\text { 4 septembre 2014 } \\
\text { 17.00-21.30 h }\end{array}$ & $\begin{array}{l}\text { Lausanne } \\
\text { World Trade } \\
\text { Center }\end{array}$ \\
\hline K23 & $\begin{array}{l}\text { Jeudi } \\
\text { 20 novembre 2014 } \\
\text { 17.00-21.30 h }\end{array}$ & $\begin{array}{l}\text { Genève } \\
\text { Crowne Plaza }\end{array}$ \\
\hline
\end{tabular}

Remise d'un cabinet médical

Le séminaire s'adresse aux médecins désirant remettre un cabinet médical. Idéalement 5-10 ans avant la remise prévue (pour des questions de taxation et prévoyance).

\section{Contenu}

- Aspects juridiques (autour du contrat de remise/reprise)

- Estimation d'un cabinet (inventaire et goodwill)

- Assurances (prévoyance, assurances à l'intérieur et autour du cabinet)

- Conséquences fiscales d'une remise.

\section{Sponsors}

Les coûts sont pris en charge par divers spon sors (voir www.fmhservices.ch).

\begin{tabular}{|c|c|c|}
\hline \multicolumn{3}{|c|}{ Dates } \\
\hline K24 & $\begin{array}{l}\text { Jeudi } \\
5 \text { juin } 2014 \\
17.00-21.30 \mathrm{~h}\end{array}$ & $\begin{array}{l}\text { Lausanne } \\
\text { World Trade } \\
\text { Center }\end{array}$ \\
\hline K25 & $\begin{array}{l}\text { Jeudi } \\
13 \text { novembre } 2014 \\
17.00-21.30 \mathrm{~h}\end{array}$ & $\begin{array}{l}\text { Genève } \\
\text { Crowne Plaza }\end{array}$ \\
\hline
\end{tabular}

Cabinet de groupe

Le séminaire s'adresse aux médecins en forma- tion voulant exercer leur future activité en cabinet de groupe et aux libres praticiens qui souhaitent affilier leur cabinet individuel à un cabinet de groupe.

\section{Contenu}

- Stratégie (objectifs du cabinet de groupe; structure de l'offre de prestations)

- Entrepreneurs (composition de l'équipe; règles de conduite; participation financière et modèles de rémunération)

- Finances \& droit (assurances, prévoyance et patrimoine; forme juridique, finances et impôts)

- Lieu d'implantation \& immobilier (exigences locales; analyse de la situation concurrentielle; aménagement du cabinet et coûts)

- Ressources humaines (qualifications; lois, règlements et contrats)

- Direction \& organisation (structure et processus; tâches, responsabilités et compétences)

- Informatique \& administration (attentes en matière de système informatique pour le cabinet; évaluation)

- Rapport d'expérience (rapport de l'expérience d'un médecin, cofondateur d'un cabinet de groupe de la région).

\section{Sponsors}

Les coûts sont pris en charge par divers sponsors (voir www.fmhservices.ch).

\begin{tabular}{|c|c|c|}
\hline at & & \\
\hline & Jeudi & Lausanne \\
\hline & $\begin{array}{l}25 \text { septembre } 2014 \\
13.30-18.00 \mathrm{~h}\end{array}$ & $\begin{array}{l}\text { World Trade } \\
\text { Center }\end{array}$ \\
\hline
\end{tabular}

Une retraite réussie

Le séminaire s'adresse aux médecins qui ne veulent pas laisser l'organisation de leur retraite (anticipée ou non) au hasard.

\section{Seminari 2014}

\section{Apertura e rilevamento di uno studio} medico

Il seminario è destinato ai medici in procinto di aprire o di rilevare uno studio medico.

\section{Contenuto}

- Business Plan (preparazione del piano di finanziamento e del credito d'esercizio, prestito bancario)

- Pianificazione (insediamento, progetto e pianificazione, scelta del mobilio, budget)

- Valutazione di uno studio medico (inventario e goodwill)

- Amministrazione di uno studio medico (interna allo studio, rapporti con la banca)

- Assicurazioni (tutte le assicurazioni necessarie interne ed esterne allo studio)
- Passaggio dallo stato di dipendente a quello di indipendente

- Fiscalità

\section{Sponsor}

Diversi sponsor si fanno carico delle spese (si rimanda al sito www.fmhservices.ch).

\section{Date}

K50

Giovedi 10 aprile $2014 \quad$ FMH Fiduciaria dalle 14.00 alle 18.00 Services

K5

$\begin{array}{ll}\text { Giovedì } & \text { Chiasso } \\ 23 \text { ottobre } 2014 & \text { FMH Fiduciaria }\end{array}$

Contenu

Ma rente va-t-elle suffire?

- Besoin éventuel de capital

- Solutions de prévoyance professionnelle

- Démarche fiscale optimalisée

Fiduciaire et prévoyance vieillesse?

- Harmonisation de la planification du départ à la retraite avec la vente du cabinet

- Economies fiscales.

Exemple pratique

- Planification de la prévoyance vieillesse.

\section{Coûts}

100 CHF (y compris documents de cours et collations).

\section{Date \\ K30 Jeudi \\ 17.00-20.00 h Center}

\section{Inscription et information}

www.fmhservices.ch ou FMH Consulting Services, Cornelia Fuchs, Burghöhe 1, 6208 Oberkirch, tél. 04192500 77, fax 0419210586.

\section{Remarque}

Les adresses des participants aux séminaires dont les coûts sont couverts en partie ou totalement par des sponsors sont communiquées aux sponsors concernés.

\section{Conditions d'annulation}

Un montant est perçu pour une absence ou une annulation. Il est de:

- 50 CHF par personne dans les 15 jours avant le début du séminaire;

- 100 CHF par personne dans les 7 jours avant le début du séminaire.

\section{Iscrizioni e informazion}

www.fmhservices.ch o FMH Consulting Services, Cornelia Fuchs, Burghöhe 1, 6208 Oberkirch, tel. 04192500 77, fax 0419210586

\section{Osservazioni}

Gli indirizzi dei partecipanti ai seminari, i cui costi sono coperti in parte o completamente da degli sponsor, vengono comunicati agli sponsor interessati.

\section{Condizioni d'annullamento}

Un importo verrà rimborsato in caso di assenza o annullamento. Esso sarà di:

- 50 CHF per persona entro i 15 giorni prima dell'inizio del seminario;

- 100 CHF per persona entro i 7 giorni prima dell'inizio del seminario. 